\title{
Clinical Interpretation of Efficacy Outcomes in Pharmacological Studies on Triple Fixed-Dose Combination Therapy for Uncontrolled Asthma: Assessment of IRIDIUM and ARGON Studies
}

\section{Paola Rogliani (ID) \\ Luigino Calzetta $\mathbb{D}^{2}$}

'Unit of Respiratory Medicine, Department of Experimental Medicine, University of Rome "Tor Vergata", Rome, Italy; ${ }^{2}$ Department of Medicine and Surgery, Respiratory Disease and Lung Function Unit, University of Parma, Parma, Italy
Correspondence: Paola Rogliani Unit of Respiratory Medicine, Department of Experimental Medicine, University of Rome "Tor Vergata", via Montpellier I, Rome, 00133, Italy Tel +390620904656

Email paola.rogliani@uniroma2.it

\begin{abstract}
The IRIDIUM and ARGON studies provided positive findings concerning the benefits of the once-daily triple mometasone furoate/indacaterol/glycopyrronium (MF/IND/ GLY) fixed-dose combination (FDC) for the treatment of uncontrolled asthma, at the least by a strict statistical point of view. In the IRIDIUM study patients received medium-dose (MD) or high-dose (HD) MF/IND/GLY or MF/IND oncex daily or HD fluticasone/salmeterol (FLU/SAL) twice daily; in the ARGON study patients received MD or HD MF/IND/GLY once daily or HD FLU/SAL twice daily + tiotropium (TIO) once daily. Since a detailed interpretation of clinical results has not yet been performed, we provided the clinical interpretation of efficacy outcomes resulting from the IRIDIUM and ARGON studies according to the currently available minimal clinically important difference (MCID) thresholds. The triple MF/IND/GLY FDC elicited beneficial clinically relevant effects compared to active comparators in asthmatic patients, according to the levels of ICS doses, by generally achieving and overcoming the MCID. The level of clinical benefit was usually greater in patients treated with HD-MF/IND/GLY compared to those treated with MD-MF/IND/GLY. Overall, HD-MF/IND/GLY induced greater clinically relevant benefits even when compared to HD-FLU/SAL + TIO. Considering that a balanced triple MF/IND/GLY FDC with MD ICS resulted as effective as HD-MF/IND in preventing moderate or severe exacerbations, thus triple ICS/LABA/LAMA FDCs with MD ICS should be considered for the treatment not only of uncontrolled asthma but also for those patients suffering from less severe forms of disease with airflow limitation as well as a possible as-needed therapeutic option.
\end{abstract}

Keywords: ARGON study, IRIDIUM study, minimal clinically important difference, MCID, triple therapy, uncontrolled asthma

\section{Background}

The interpretation of findings obtained from randomized controlled trials (RCTs) is traditionally based on the statistical significance of results rather than clinical relevance, usually reported as the minimal clinically important difference (MCID). ${ }^{1}$ The concept of MCID in RCTs has been known for a long time, and it can be defined as the smallest treatment effect that patients perceive as beneficial and which would mandate, in the absence of serious adverse events and excessive cost, a change in the patient's management. ${ }^{2}$ In this regard, it has been shown that in papers reporting data from pharmacological RCTs the clinical importance of 
results is often not adequately discussed or the authors do not provide sufficient information to allow readers makeing their own interpretation. ${ }^{1}$

The $\mathrm{P}$ value is probably a statistical concept associated with most fallacies and misuses in pharmacological studies, as a common misconception is that in positive RCTs the outcome simply needs to be statistically significant. ${ }^{3}$ Along with statistically significance testing, the investigated outcome requires careful interpretation of their potential clinical impact.

The once-daily inhaled corticosteroid/long-acting $\beta_{2}$ agonist/long-acting muscarinic antagonist (ICS/LABA/ LAMA) fixed-dose combination (FDC) mometasone furoate/indacaterol/glycopyrronium (MF/IND/GLY) has been recently approved for the treatment of asthma. The current Global Initiative for Asthma (GINA, 2021) ${ }^{4}$ document recommends to use this triple FDC as controller option at asthma Step 4 and as preferred treatment at asthma Step 5 in adults and adolescents according to the positive results and significant benefits for patients resulting from the Phase III RCTs IRIDIUM ${ }^{5}$ and ARGON. ${ }^{6}$

In the IRIDIUM study, ${ }^{5}$ patients with inadequately controlled asthma received medium-dose (MD) or highdose (HD) MF/IND/GLY or MF/IND once daily or HD fluticasone/salmeterol (FLU/SAL) twice daily. In the ARGON study, ${ }^{6}$ patients with uncontrolled asthma received MD or $\mathrm{HD} \mathrm{MF} / \mathrm{IND} / \mathrm{GLY}$ once daily or HD FLU/SAL twice daily + tiotropium (TIO) once daily. The level of ICS doses (MD and HF) included in the combinations is ranked according to the current GINA recommendations ${ }^{4}$ and the National Institute for Health and Care Excellence (NICE) ${ }^{7}$ guidelines.

Although both these studies provided positive findings at the least by a statistical point of view, ${ }^{6,8}$ to date a detailed interpretation of clinical results has not yet been performed. Therefore, here we provide the clinical interpretation of efficacy outcomes resulting from the IRIDIUM $^{5}$ and $\mathrm{ARGON}^{6}$ studies according to the currently available MCIDs.

\section{Spirometry}

\section{FEV ,}

Clinical studies in asthmatic patients often include forced expiratory volume in $1 \mathrm{~s}\left(\mathrm{FEV}_{1}\right)$ as a primary outcome since it has been generally recognized by research community and regulatory agencies to be a suitable variable to assess airflow obstruction. ${ }^{9}$ The current standards of
MCID threshold for $\mathrm{FEV}_{1}$ have been proposed for comparisons vs. placebo or for improvements vs. baseline, ${ }^{9-12}$ but difficulty still remains in evaluating the clinical significance of spirometric outcomes when comparing either active treatments or drugs administered as triple FDCs vs. the same agents administered as dual FDCs. ${ }^{13}$ Therefore, when assessing $\mathrm{FEV}_{1}$ in chronic obstructive respiratory disorders, it has been suggested a threshold MCID value of $>100 \mathrm{~mL}$ for trough $\mathrm{FEV}_{1}$ for comparisons between active treatments and either placebo or baseline, ${ }^{10,12}$ whereas the level of MCID threshold should be $>60 \mathrm{~mL}$ for trough $\mathrm{FEV}_{1}$ when active treatments are compared to each other. ${ }^{13}$

In the IRIDIUM study, ${ }^{5}$ MD-MF/IND/GLY significantly improved trough forced $\mathrm{FEV}_{1}$ by $76 \mathrm{~mL}(95 \% \mathrm{CI}$ 41-111) vs. MD-MF/IND ( $\mathrm{P}<0.001)$ and $\mathrm{HD}-\mathrm{MF} / \mathrm{IND} /$ GLY significantly improved trough $\mathrm{FEV}_{1}$ by $65 \mathrm{~mL}$ (95\% CI 31-99) vs. HD-MF/IND ( $<<0.001)$. Similarly, $\mathrm{MD}-\mathrm{MF} / \mathrm{IND} / \mathrm{GLY}$ significantly improved trough $\mathrm{FEV}_{1}$ by $99 \mathrm{~mL}(95 \%$ CI $64-133)$ vs. HD-FLU/SAL $(\mathrm{P}<0.001)$ and HD-MF/IND/GLY significantly improved trough $\mathrm{FEV}_{1}$ by $119 \mathrm{~mL}$ (95\% CI 85-154) vs. HD-FLU/SAL $(\mathrm{P}<0.001) . \mathrm{MF} / \mathrm{IND} / \mathrm{GLY}$ administered at both ICS doses reached and overcame the MCID for trough $\mathrm{FEV}_{1}$ when comparing active treatments (MCID $>60 \mathrm{~mL}$ ). Of note, when MF/IND/GLY was compared to HD-FLU/SAL, the triple FDC reached the MCID for trough $\mathrm{FEV}_{1}$ with respect to placebo or baseline (MCID $>100 \mathrm{~mL}$ ).

In the ARGON study, ${ }^{6} \mathrm{HD}-\mathrm{MF} / \mathrm{IND} / \mathrm{GLY}$ significantly improved trough $\mathrm{FEV}_{1}$ by $96 \mathrm{~mL}(95 \%$ CI 46-146) vs. HD-FLU/SAL + TIO $(\mathrm{P}<0.001)$, and it reached and overcame the MCID for trough $\mathrm{FEV}_{1}$ when compared to active treatments (MCID $>60 \mathrm{~mL}$ ).

\section{PEF}

To date, no studies report a certain MCID threshold for peak expiratory flow (PEF) ${ }^{14}$ however a minimal patient perceivable improvement (MPPI) value of $5.39 \%$ has been proposed for PEF as difference between active treatment groups. $^{12}$

In the ARGON study, ${ }^{6} \mathrm{HD}-\mathrm{MF} / \mathrm{IND} / \mathrm{GLY}$ significantly $(\mathrm{P}<0.01)$ improved morning PEF vs. HD-FLU/SAL + TIO (35.85 L/min vs $26.29 \mathrm{~L} / \mathrm{min}$ ); the absolute treatment difference was $9.56 \mathrm{~L} / \mathrm{min}$ (95\% CI 2.89-6.29) and the percentage treatment difference was $26.67 \%$. HD-MF/ IND/GLY also significantly $(\mathrm{P}<0.01)$ improved evening PEF vs. HD-FLU/SAL + TIO (31.86 L/min and $22.71 \mathrm{~L} /$ min); the absolute treatment difference was $9.15 \mathrm{~L} / \mathrm{min}$ 
(95\% CI 2.57-15.72) and the percentage treatment difference was $28.72 \%$. Thus, it seems that HD-MF/IND/GLY reached and overcame the MPPI for PEF when compared to HD-FLU/SAL + TIO.

\section{Exacerbations}

Exacerbations have negative impact on quality of life of asthmatic patients. Since severe exacerbations may represent life-threatening events, preventing even a single episode could be considered a minimal clinically important improvement. In any case, the MCID threshold for severe asthma exacerbations has been identified as a reduction $>20 \%$ in annual exacerbation rate. ${ }^{9}$

In the IRIDIUM study, ${ }^{5}$ HD-MF/IND/GLY significantly $(\mathrm{P} \leq 0.05)$ reduced the risk of all exacerbations (mild, moderate, and severe) and severe exacerbations vs. HD-MF/IND (rate ratio: 0.79 [95\% CI 0.66-0.96] and 0.78 [95\% CI 0.61-1.00], respectively) by reaching and overcoming the MCID for exacerbations ( $-21 \%$ and $-22 \%$, respectively). When compared to HD-FLU/SAL, HD-MF/ IND/GLY significantly $(\mathrm{P}<0.001)$ reduced in a clinically relevant manner the risk of moderate or severe exacerbations (rate ratio: 0.64 [95\% CI 0.52-0.78]; percentage reduction: $-36 \%$ ), severe exacerbations (rate ratio: 0.58 [95\% CI 0.45-0.73]; percentage reduction: $-42 \%$ ), and all exacerbations (mild, moderate, and severe; rate ratio: 0.60 [95\% CI 0.50-0.72]; percentage reduction: $-40 \%$ ). MD-MF/IND/GLY reduced in a significant $(\mathrm{P}<0.001)$ and clinically relevant manner vs. HD-FLU/SAL the risk of all exacerbations (mild, moderate, and severe; rate ratio: 0.70 [95\% CI 0.58-0.84]; percentage reduction: $-30 \%$ ); MD$\mathrm{MF} / \mathrm{IND} / \mathrm{GLY}$ also significantly reduced $(\mathrm{P}<0.05)$ the risk of moderate or severe exacerbations (rate ratio: 0.81 [95\% CI 0.66-0.99]) vs. HD-FLU/SAL, but the percentage of reduction $(-19 \%)$ was borderline with the MCID for exacerbations.

In the ARGON study, ${ }^{6} \mathrm{HD}-\mathrm{MF} / \mathrm{IND} / \mathrm{GLY}$ significantly reduced $(\mathrm{P} \leq 0.05)$ the risk of moderate exacerbations (rate ratio: 0.57 [95\% CI 0.33-0.98]) vs. HD-FLU/SAL + TIO, by reaching and overcoming the MCID for exacerbations $(-43 \%)$.

\section{Questionnaires ACQ-7}

The MCID for Asthma Control Questionnaire 7 (ACQ-7) has been well established and accepted as 0.5 points. ${ }^{9,10,14}$
In the IRIDIUM study, ${ }^{5} \mathrm{MF} / \mathrm{IND} / \mathrm{GLY}$ administered at both ICS doses reached and reduced almost two-fold the MCID for ACQ-7 vs. baseline (MD-MF/IND/GLY: -0.97 ; HD-MF/IND/GLY: -0.97$)$, and a significantly $(\mathrm{P}<0.05)$ greater proportion of patients treated with HD-MF/IND/ GLY achieved the MCID for AQLQ vs. HD-FLU/SAL $(+8.2 \%)$. Moreover, $\mathrm{MF} / \mathrm{IND} / \mathrm{GLY}$ administered at both ICS doses significantly $(\mathrm{P}<0.05)$ reduced ACQ-7 vs. HDFLU/SAL (MD-MF/IND/GLY: -0.08; HD-MF/IND/ GLY: -0.09 ).

In the ARGON study, ${ }^{6}$ the effect of HD-MF/IND/GLY on ACQ-7 was significantly $(\mathrm{P}<0.01)$ greater than that elicited by HD-FLU/SAL + TIO (delta effect: -0.12 [95\% CI $-0.22--0.03]$ ), and MF/IND/GLY administered at both ICS doses reached and reduced more than two-fold the MCID for ACQ-7 vs. baseline (MD-MF/IND/GLY: -1.08; HD-MF/IND/GLY: -1.172).

\section{AQLQ}

The MCID for Quality of Life Questionnaire (AQLQ) has been determined to be a cut point of 0.5.9,14

In the ARGON study, ${ }^{6}$ a significantly $(\mathrm{P}<0.05)$ greater proportion of patients treated with $\mathrm{HD}-\mathrm{MF} / \mathrm{IND} / \mathrm{GLY}$ achieved the MCID for AQLQ vs. HD-FLU/SAL + TIO $(+8.1 \%) .{ }^{9}$ Moreover, MF/IND/GLY administered at both ICS doses reached and overcome the MCID for AQLQ vs. baseline (MD-MF/IND/GLY: $\quad-0.72$; HD-MF/IND/ GLY: -0.83).

\section{SGRQ}

The MCID for St. George's Respiratory Questionnaire (SGRQ) corresponds to a decrease of $>4$ points. $^{9}$

In the ARGON study, ${ }^{6}$ the effect of HD-MF/IND/GLY on SGRQ was significantly $(\mathrm{P}<0.05)$ greater than that elicited by HD-FLU/SAL + TIO (delta effect: -2.00 [95\% CI -3.90 - -0.09]). Moreover, MF/IND/GLY administered at both ICS doses reached and reduced around three-fold the MCID for SGRQ vs. baseline (MD-MF/ IND/GLY: -11.95; HD-MF/IND/GLY: -13.29).

\section{Conclusion}

The assessment of efficacy outcomes reported in the IRIDIUM $^{5}$ and ARGON $^{6}$ studies indicates that the triple $\mathrm{MF} / \mathrm{IND} / \mathrm{GLY}$ FDC elicits beneficial clinically relevant effects compared to active comparators in asthmatic patients, according to the levels of ICS doses as shown in Table 1, where the dose of MF was increased from 80 $\mu \mathrm{g}$ to $160 \mu \mathrm{g}$. As expected, the level of clinical benefit 
Table I Clinical effect of MF/IND/GLY FDC administered at different ICS doses compared to active comparators on efficacy outcomes in asthmatic patients as reported in the IRIDIUM ${ }^{5}$ and ARGON $^{6}$ studies

\begin{tabular}{|c|c|c|c|c|c|}
\hline Outcome & Treatment & Active comparator & Delta & $\begin{array}{l}\text { Suggested } \\
\text { MCID }\end{array}$ & $\begin{array}{l}\text { Beneficial clinically } \\
\text { relevant effect }\end{array}$ \\
\hline \multirow[t]{5}{*}{$\mathrm{FEV}_{\text {। }}$} & MD-MF/IND/GLY & MD-MF/IND & $76 \mathrm{~mL}$ & \multirow[t]{5}{*}{$>60 \mathrm{~mL}^{*}$} & Yes \\
\hline & MD-MF/IND/GLY & HD-FLU/SAL & $99 \mathrm{~mL}$ & & Yes \\
\hline & HD-MF/IND/GLY & HD-MF/IND & $65 \mathrm{~mL}$ & & Yes \\
\hline & HD-MF/IND/GLY & HD-FLU/SAL & $119 \mathrm{~mL}$ & & Yes \\
\hline & HD-MF/IND/GLY & HD-FLU/SAL + TIO & $96 \mathrm{~mL}$ & & Yes \\
\hline Morning PEF & HD-MF/IND/GLY & HD-FLU/SAL + TIO & $26.67 \%$ & \multirow[t]{2}{*}{$>5.39 \%^{\#}$} & Yes \\
\hline Evening PEF & HD-MF/IND/GLY & HD-FLU/SAL + TIO & $28.72 \%$ & & Yes \\
\hline \multirow{3}{*}{$\begin{array}{l}\text { All exacerbations (mild, } \\
\text { moderate, and severe) }\end{array}$} & MD-MF/IND/GLY & HD-FLU/SAL & $-30 \%$ & \multirow[t]{8}{*}{$>-20 \%$} & Yes \\
\hline & HD-MF/IND/GLY & HD-MF/IND & $-21 \%$ & & Yes \\
\hline & HD-MF/IND/GLY & HD-FLU/SAL & $-40 \%$ & & Yes \\
\hline \multirow{2}{*}{$\begin{array}{l}\text { Moderate or severe } \\
\text { exacerbations }\end{array}$} & MD-MF/IND/GLY & HD-FLU/SAL & $-19 \%$ & & Borderline \\
\hline & HD-MF/IND/GLY & HD-FLU/SAL & $-36 \%$ & & Yes \\
\hline $\begin{array}{l}\text { Moderate } \\
\text { exacerbations }\end{array}$ & HD-MF/IND/GLY & HD-FLU/SAL + TIO & $-43 \%$ & & Yes \\
\hline \multirow[t]{2}{*}{ Severe exacerbations } & HD-MF/IND/GLY & HD-MF/IND & $-22 \%$ & & Yes \\
\hline & HD-MF/IND/GLY & HD-FLU/SAL & $-42 \%$ & & Yes \\
\hline ACQ-7 & HD-MF/IND/GLY & HD-FLU/SAL & $+8.2 \%$ responders & $>0.5$ points & $\begin{array}{c}\text { A greater proportion of } \\
\text { patients achieved the MCID }\end{array}$ \\
\hline AQLQ & HD-MF/IND/GLY & HD-FLU/SAL + TIO & $+8.1 \%$ responders & $>0.5$ points & $\begin{array}{c}\text { A greater proportion of } \\
\text { patients achieved the MCID }\end{array}$ \\
\hline SGRQ & HD-MF/IND/GLY & HD-FLU/SAL + TIO & -2.00 & $>4$ units & No \\
\hline
\end{tabular}

Notes: * vs. active treatment. ${ }^{*}$ Assessed via MPPI.

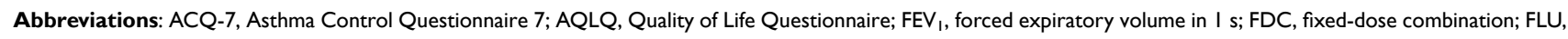
fluticasone; GLY, glycopyrronium; HD, high-dose; ICS, inhaled corticosteroid; IND, indacaterol; MD, medium-dose; MCID, minimal clinically important difference; MF, mometasone furoate; MPPI, minimal patient perceivable improvement; PEF, peak expiratory flow; SAL, salmeterol; SGRQ, St. George's Respiratory Questionnaire; TIO, tiotropium.

was generally greater in patients treated with $\mathrm{HD}-\mathrm{MF} /$ IND/GLY compared to those treated with MD-MF/IND/ GLY. Interestingly, the current evidence indicates that HD-MF/IND/GLY induced greater clinically relevant benefits on $\mathrm{FEV}_{1}, \mathrm{PEF}$, moderate exacerbations and AQLQ even when compared to HD-FLU/SAL + TIO, a free triple combination therapy including an ICS administered at high dose. However, according to the taxonomy suggested by Jones ${ }^{15}$ to rank the impact of treatments on SGRQ, the beneficial effect of HD-MF/ IND/GLY on SGRQ was statistically but not clinically significant when compared to HD-FLU/SAL + TIO.
Indeed, these data fully support the position of the European Medicine Agency (EMA) reported in the "Human Medicines Highlights 2020" document, which included the triple MF/IND/GLY FDC (Enerzair Breezhaler) among a selection of medicines approved in 2020 , and the only one in the therapeutic area of respiratory medicine, that represents an outstanding contribution to public health. ${ }^{16}$ Certainly, as stated in the EMA document, ${ }^{16}$ the authorisation of new medicines such as the triple MF/IND/GLY FDC is essential to advancing public health as it brings new opportunities to treat patients with uncontrolled asthma. 
Finally, but not less important, a balanced triple MF/IND/GLY FDC with MD ICS resulted as effective as HD-MF/IND in preventing moderate or severe exacerbations, ${ }^{5,8}$ a phenomenon that was detected also against the increased contractile tone of human hyperresponsive bronchi and airway inflammation. ${ }^{17}$ Thus, triple ICS/LABA/LAMA FDCs with moderate ICS dose should be considered for the treatment not only of uncontrolled asthma but also for those patients suffering from less severe forms of disease with airflow limitation as well as a possible as-needed therapeutic option.

\section{Disclosure}

Professor Paola Rogliani reports grants, personal fees, non-financial support from Novartis, outside the submitted work. Dr Luigino Calzetta reports grants, personal fees from Novartis, outside the submitted work. The authors report no other conflicts of interest in this work.

\section{References}

1. Chan KBY, Man-Son-Hing M, Molnar FJ, et al. How well is the clinical importance of study results reported? An assessment of randomized controlled trials. Can Med Assoc J. 2001; 165:1197.

2. Jaeschke R, Singer J, Guyatt GH. Measurement of health status: ascertaining the minimal clinically important difference. Control Clin Trials. 1989;10(4):407-415. doi:10.1016/0197-2456(89)900 05-6

3. Calzetta L, Ritondo BL, Coppola A, et al. Factors influencing the efficacy of COVID-19 vaccines: a quantitative synthesis of Phase III trials. Vaccines. 2021;9:341. doi:10.3390/vaccines904 0341

4. GINA. 2021 GINA main report | Global Initiative for Asthma [Internet]; 2021 [cited July 22, 2021]. Available from: https:// ginasthma.org/wp-content/uploads/2021/05/GINA-Main-Report-2021V2-WMS.pdf. Accessed December 29, 2021.
5. Kerstjens HAM, Maspero J, Chapman KR, et al. Once-daily, singleinhaler mometasone-indacaterol-glycopyrronium versus mometasone-indacaterol or twice-daily fluticasone-salmeterol in patients with inadequately controlled asthma (IRIDIUM): a randomised, double-blind, controlled Phase 3 study. Lancet Respir Med. 2020;8:1000-1012. doi:10.1016/S2213-2600(20)30190-9

6. Gessner C, Kornmann O, Maspero J, et al. Fixed-dose combination of indacaterol/glycopyrronium/mometasone furoate once-daily versus salmeterol/fluticasone twice-daily plus tiotropium once-daily in patients with uncontrolled asthma: a randomised, Phase IIIb, noninferiority study (ARGON). Respir Med. 2020;170:106021. doi:10.1016/j.rmed.2020.106021

7. NICE. National Institute for Health and Care Excellence (NICE). Inhaled corticosteroid doses for NICE's asthma guideline; 2018. Available from: www.nice.org.uk/guidance/ng80/resources/inhaledcorticosteroid-doses-pdf-4731528781. Accessed January 16, 2020.

8. Virchow JC. Assessing the benefits of triple versus dual fixed-dose combinations for the treatment of severe asthma. Lancet Respir Med. 2020;8:937-939. doi:10.1016/S2213-2600(20)30303-9

9. Bonini M, Di Paolo M, Bagnasco D, et al. Minimal clinically important difference for asthma endpoints: an expert consensus report. Eur Respir Rev. 2020;29:1-14. doi:10.1183/16000617.0137-2019

10. Parulekar A, Alobaidy A, Hanania N. Asthma outcomes revisited. Curr Opin Pulm Med. 2013;19:6-12. doi:10.1097/MCP.0b013e 32835b11f6

11. Pellegrino R, Viegi G, Brusasco V, et al. Interpretative strategies for lung function tests. Eur Respir J. 2005;26:948-968. doi:10.1183/ 09031936.05 .00035205

12. Santanello N, Zhang J, Seidenberg B, et al. What are minimal important changes for asthma measures in a clinical trial? Eur Respir J. 1999;14:23-27. doi:10.1034/j.1399-3003.1999.14a06.x

13. Bateman E. Lack of clinically relevant differences between combination therapy and monotherapy in COPD. Eur Respir J. 2014;43:1204-1205. doi:10.1183/09031936.00156313

14. Validity of outcome measures; 2016 [cited July 20, 2021]. Available from: https://www.ncbi.nlm.nih.gov/books/NBK409867/. Accessed December 29, 2021.

15. Jones PW. St. George's respiratory questionnaire: MCID. COPD. 2005;2:75-79. doi:10.1081/COPD-200050513

16. Human medicines: highlights of 2020 | European Medicines Agency [Internet]; [cited July 20, 2021]. Available from: https:/www.ema. europa.eu/en/news/human-medicines-highlights-2020. Accessed December 29, 2021.

17. Rogliani P, Ritondo BL, Facciolo F, et al. Indacaterol, glycopyrronium, and mometasone: pharmacological interaction and anti-inflammatory profile in hyperresponsive airways. Pharmacol Res. 2021;172:105801. doi:10.1016/j.phrs.2021.105801
The Journal of Experimental Pharmacology is an international, peerreviewed, open access journal publishing original research, reports, reviews and commentaries on all areas of laboratory and experimental pharmacology. The manuscript management system is completely online and includes a very quick and fair peer-review system. Visit http://www.dovepress.com/testimonials.php to read real quotes from published authors. 